\title{
Recognition of Mitochondrial Proteins in Plasmodium Based on the Tripeptide Composition
}

OPEN ACCESS

Edited by:

Liang Cheng,

Harbin Medical University, China

Reviewed by:

Junwei Han,

Harbin Medical University, China

Ying Wang,

Xiamen University, China

${ }^{*}$ Correspondence:

Juan Wang

wangjuan@imu.edu.cn

Specialty section:

This article was submitted to

Molecular Medicine

a section of the journal

Frontiers in Cell and Developmental

Biology

Received: 01 July 2020

Accepted: 13 August 2020

Published: 16 September 2020

Citation:

Bian H, Guo M and Wang J (2020) Recognition of Mitochondrial Proteins

in Plasmodium Based on the

Tripeptide Composition

Front. Cell Dev. Biol. 8:578901.

doi: 10.3389/fcell.2020.578901

\author{
Haodong Bian ${ }^{1}$, Maozu Guo ${ }^{2,3}$ and Juan Wang 1,4* \\ ${ }^{1}$ School of Computer Science, Inner Mongolia University, Hohhot, China, ${ }^{2}$ School of Electrical and Information Engineering, \\ Beijing University of Civil Engineering and Architecture, Beijing, China, ${ }^{3}$ Beijing Key Laboratory of Intelligent Processing for \\ Building Big Data, Beijing, China, ${ }^{4}$ Stage Key Laboratories of Reproductive Regulation \& Breeding of Grassland Livestock, \\ Hohhot, China
}

Mitochondria play essential roles in eukaryotic cells, especially in Plasmodium cells. They have several unusual evolutionary and functional features that are incredibly vital for disease diagnosis and drug design. Thus, predicting mitochondrial proteins of Plasmodium has become a worthwhile work. However, existing computational methods can only predict mitochondrial proteins of Plasmodium falciparum ( $P$. falciparum for short), and these methods have low accuracy. It is highly desirable to design a classifier with high accuracy for predicting mitochondrial proteins for all Plasmodium species, not only P. falciparum. We proposed a novel method, named as PM-OTC, for predicting mitochondrial proteins in Plasmodium. PM-OTC uses the Support Vector Machine (SVM) as the classifier and the selected tripeptide composition as the features. We adopted the 5-fold cross-validation method to train and test PM-OTC. Results demonstrate that PM-OTC achieves an accuracy of $94.91 \%$, and performances of PM-OTC are superior to other methods.

Keywords: malaria, Plasmodium, mitochondrion, tripeptide composition, support vector machine

\section{INTRODUCTION}

The parasite Plasmodium is the main cause of malaria, and kills more than one million African children annually (Phillips et al., 2017). There are approximately $40 \%$ humans whose are infected by malaria in the world. Four species of Plasmodium that can infect humans with malaria are $P$. falciparum, Plasmodium vivax, Plasmodium malaria, and Plasmodium ovale, respectively, where P. falciparum is the most lethal (Gardner et al., 2002). Research on the mitochondrial evolution and functions of Plasmodium indicates that Plasmodium mitochondrion is suitable targets for anti-parasitic drugs (Vaidya and Mather, 2009). Thus, it is exceptionally important to predict mitochondrial proteins of Plasmodium.

Traditional methods for predicting protein functions are based on biological experiments and they are costly and time-consuming. So, the researchers proposed the computational methods to predict the protein functions (Wei et al., 2015, 2017; Qu et al., 2019). The machine learning algorithms can achieve the prediction (Zou, 2019). There are two main steps of the machine learning: classifier and feature. For the classifiers, Logistic Regression (LR) has been used to recognize protein subcellular localization (Wan et al., 2015). Naïve Bayes (NB, Rish, 2001) is applied to predict protein-protein interaction sites (Murakami and Mizuguchi, 2010). As an efficient and powerful machine learning algorithm, SVM (Vapnik, 2000) has been applied widely for predicting protein subcellular localization (Hua and Sun, 2001; Kumar et al., 2018), G-protein coupled 
receptors (Karchin et al., 2002; Bhasin and Raghava, 2004a), protein-protein interactions prediction (Guo et al., 2008), and protein fold recognition (Ding and Dubchak, 2001).

For the features, there are many methods for extracting features from the protein primary sequences. The methods for extracting features of the amino acid, dipeptide and tripeptide from protein sequences can generate fixed-length data for the protein sequences with different length. Nakashima and Nishikawa (1994) first proposes the amino acid composition (AAC) with 20 dimensional vectors, i.e., the frequency of each single amino acid, to represent proteins. Dipeptide composition (DPC) with 400 dimensional vectors, i.e., the frequency of each one pair of amino acids in protein sequences, is used to discriminate protein subcellular localization (Bhasin and Raghava, 2004b; Ahmad et al., 2016). Furthermore, the researchers propose 8000 dimensional tripeptide composition (TPC) based on the structural properties of proteins, which has been used to predict protein subcellular localization (Liao et al., 2011) and sub-chloroplast localization (Lin et al., 2013). Besides, split amino acid composition (SAAC), which divides a whole sequence into three parts: N-terminal, C-terminal, and a region between the two, i.e., the remaining amino acids between N- and C-terminal, is introduced (Chou, 2005) and used for mitochondrial proteins prediction (Kumar et al., 2006, 2018). Additionally, Shen and Chou (2006) proposes pseudo amino acid composition (PseAAC) based on the physicochemical characteristics of proteins. The researchers used the PSI-BLAST software to compute the position-specific score matrices (PSSM) for extracting evolutionary information from protein sequences (Altschul et al., 1997). And PSSM also has been used for proteins subcellular localization prediction (Rashid et al., 2007) and protein-protein interaction site prediction (Zeng et al., 2019).

There are lots of approaches for predicting mitochondrial proteins of P. falciparum. Bender et al. (2003) uses the neural network and the relative amino acid frequency to predict mitochondrial transit peptides (mTPs) of P. falciparum. Verma et al. (2010) applies the SVM to predict mitochondrial proteins of $P$. falciparum and uses SAAC and PSSM to represent protein sequences. Jia et al. (2011) uses Bi-profile Bayes and SAAC to extract protein sequence features and uses the SVM classifier as the classifier to train two models (PfMP-N25 and PfMP-30) in order to recognize mitochondrial proteins of $P$. falciparum. Chen et al. (2012) proposes an increment of diversity (ID) method based on the n-peptide composition of the reduced amino acid alphabet (RAAA) to predict mitochondrial proteins of P. falciparum. Furthermore, Ding and Li (2015) uses the analysis of variance (ANOVA) to reduce the feature dimension and generate the optimal 5-gap dipeptide composition from the protein sequences. However, all these methods mentioned above are used to predict only mitochondrial proteins of $P$. falciparum. Our paper will introduce a noval model, named by PM-OTC, to predict mitochondrial proteins of all Plasmodium. PM-OTC uses the SVM to classify and selects several tripeptides to represent the proteins. To evaluate the performance of PM-OTC, we adopted 5 -fold cross-validation to train and test our method on the two datasets: the PM275 (collected from UniprotKB/SwissProt) and the PfM175 (used by Bender et al., 2003).

\section{MATERIALS AND METHODS}

\subsection{Datasets}

\subsubsection{PM275}

The proteins of PM275 are selected from UniprotKB/SwissProt (released 2020_01) by the following rules: (1) without ambiguous amino acids, such as "B," "X," and "Z;" (2) their function that have been confirmed by biological experiments; (3) sequences with $>50$ length. Here we obtain 54 mitochondrial proteins as positive examples, and 340 non-mitochondrial proteins as negative examples, including cytosol proteins, secreted proteins, and apicoplast proteins. Next we used the CD-HIT (Fu et al., 2012) software with global alignment and sequence identity threshold set to 0.4 to process negative sequences in order to eliminate the similar sequences. Then the protein sequences whose sequence similarity is more than or equal to $40 \%$ are regarded as the same cluster and the longest sequence from each cluster are chosen the resulting sequences. Finally, we obtained 221 non-mitochondrial proteins as the negative examples. So, the PM275 contains 54 mitochondrial proteins and 221 nonmitochondrial proteins.

\subsubsection{PfM175}

The PfM175 is mainly used in predict the mitochondrial proteins of $P$. falciparum. This dataset includes 40 mitochondrial proteins and 135 non-mitochondrial proteins (61 cytoplasmic, 21 secretory, and 53 apicoplast, respectively).

\subsection{Sequences Representation}

A protein sequence needs an efficient mathematical representation that can correctly express the inherent connection with the prediction types. To efficiently identify mitochondrial proteins of Plasmodium and build a robust model, we synthetically considered three sequences of features based on the protein primary sequence.

\subsubsection{AAC}

AAC has low complexity and has been widely used to predict the function of proteins. Given a protein sequence $S$ with $L$ residue, $\mathrm{AAC}$ represents it as following:

$$
A A C(S)=\left(f_{1}, f_{2}, \cdots, f_{20}\right)^{T}
$$

where $f_{i}=n_{i} / L(i=1,2, \cdots, 20), n_{i}$ is the frequency of the $i$-th amino acid.

\subsubsection{DPC}

DPC computes the frequency of two amino acids. A protein sequence can be represented by a 400 dimensional vector. DPC contains information about the proportion of amino acids as well as the order of sequence.

$$
\begin{gathered}
D P C(S)=\left(f_{1}, f_{2}, \cdots, f_{400}\right)^{T} \\
f_{i}=\frac{\operatorname{dep}(i)}{\sum \operatorname{dep}(i)}
\end{gathered}
$$


where $\operatorname{dep}(i)$ is one out of 400 dipeptides, $\sum \operatorname{dep}(i)$ represents total number of all possible dipeptides in sequence $S$.

\subsubsection{TPC}

TPC computes the frequency of three contiguous amino acids. A protein sequence can be represented by a 8000 dimensional vector.

$$
\begin{gathered}
\operatorname{TPC}(S)=\left(f_{1}, f_{2}, \cdots, f_{8000}\right)^{T} \\
f_{i}=\frac{\operatorname{tep}(i)}{\sum \operatorname{tep}(i)}
\end{gathered}
$$

where tep $(i)$ is one out of 8000 tripeptides, $\sum$ tep $(i)$ represents total number of all possible tripeptides in sequence $S$.

\subsection{Support Vector Machine}

SVM is a powerful and efficient machine learning algorithm for linear, non-linear classification and regression. Compared with other machine learning algorithms, the advantage of the SVM algorithm is that the dimension of SVM parameters equals the number of training samples (Zavaljevski et al., 2002).

SVM algorithm aims to calculate an optimal hyperplane that can separate two samples correctly in space. The optimal hyperplane, also known as support vector, is a set of vectors obtained by maximizing the separating margin on the training set. For linear separable classification problems, the optimal hyperplane can be directly obtained by the constrained optimization problem. For non-linear classification, the advantage of SVM is to introduce kernel function and transform the non-linear classification problem into a linear classification problem (Amari and Wu, 1999; Hofmann et al., 2008). The essence of kernel function or kernel technique is to map Euclidean space to Hilbert space by non-linear transformation so that the non-linear classification problem of original space can be transformed into the linear classification problem of calculating the optimal hyperplane in high dimensional space. Scikit-learn (Pedregosa et al., 2011), which provides linear, Gaussian (RBF), polynomial, and sigmoid kernel function, are adopted to implement the SVM classifier. We mainly use linear kernel function and Gaussian kernel function in our experiments. In order to find the optimal values of the two parameters $\mathrm{C}$ and $\gamma$, we employ a grid search method with 5 -fold cross-validation. The range of $\mathrm{C}$ and $\gamma$ are $\left[2^{-1}, 2^{3}\right]$ and $\left[2^{-4}, 2^{-1}\right]$ with the step of 2 .

\subsection{Feature Selection}

TPC can obtain 8000 feature values for a protein. However, these feature values may contain redundant and noisy information which will affect the training model and can lead to low prediction accuracy eventually. Accordingly, it is vital to select appropriate features from TPC to improve the prediction accuracy. The analysis of variance (ANOVA) can filter out the tripeptides with low variance, which is suitable for processing TPC because of its lots of zero values. ANOVA can compute the difference in the mean of two or more samples. ANOVA computes a F-value by the difference within the same group and the difference among different groups (Anderson, 2001). The F-value for the $\xi$-th tripeptide is defined as:

$$
F(\xi)=\frac{s_{B}^{2}(\xi)}{s_{W}^{2}(\xi)}
$$

where $\mathrm{Sb} 2$ and Sw2 are calculated by the following formulas:

$$
\begin{aligned}
& s_{B}^{2}(\xi)=\sum_{i=1}^{K} m_{i}\left(\frac{\sum_{j=1}^{m_{i}} f_{\xi}(i, j)}{m_{i}}-\frac{\sum_{i=1}^{K} \sum_{j=1}^{m_{i}} f_{\xi}(i, j)}{\sum_{i=1}^{K} m_{i}}\right)^{2} /(K-1) \\
& s_{W}^{2}(\xi)=\sum_{i=1}^{K} \sum_{j=1}^{m_{i}}\left(f_{\xi}(i, j)-\frac{\sum_{i=1}^{K} \sum_{j=1}^{m_{i}} f_{\xi}(i, j)}{\sum_{i=1}^{K} m_{i}}\right)^{2} /(M-K)
\end{aligned}
$$

where $\mathrm{K}$ and $\mathrm{M}$ represent the number of groups and total number of samples. $f_{\xi}(i, j)$ is the frequency of the $\xi$-th tripeptide of the $j$-th sample in the $i$-th group. $m_{i}$ represents the number of samples in the $i$-th group. The $F(\xi)$ in Equation (6) computes the ratio of the sample variance among groups and the sample variance within groups. MSB (mean square between) denotes the sample variance between groups and MSW (mean square within) denotes the sample variance within groups. If the value of $F(\xi)$ is away from 1, then there is a significant difference between MSB and MSW. On the contrary, if the value of $F(\xi)$ is close to 1 , then there is no significant difference between MSB and MSW. The larger the $F(\xi)$ value is, the greater the impact of the $\xi$-th tripeptide on the predicted results. So we rank 8000 tripeptides in the descending order of $F(\xi)$ values and employ an Incremental Feature Selection (IFS) strategy to find the optimized TPC with the highest prediction accuracy as the features. The detailed steps are as follows. First, we choose the tripeptide with the highest Fvalue to generate an initial feature set. Second, we select another tripeptide with the second-highest F-value and add it to the initial feature set and form a new feature set. And repeat this step to form 8000 feature sets. Each feature set is used to train and test a prediction model. Finally, we choose the feature set, which prediction model based on it achieve the maximum accuracy, as the optimized TPC.

\subsection{Performance Measures}

We use 5-fold cross-validation to assess the prediction performance of our method. First, we randomly divide the dataset into five mutually exclusive subsets of similar size. Second, we choose one subset as the testing dataset and the other four subsets as the training dataset. So, we run five times of training and testing, and return the average value of five test results.

Here, six metrics for evaluating methods are used, accuracy, sensitivity, precision, recall, F-score, and the Matthews correlation coefficient (MCC), respectively. The detailed formulas are followings:

$$
\text { Accuracy }=\frac{\mathrm{TP}+\mathrm{TN}}{\mathrm{TP}+\mathrm{TN}+\mathrm{FP}+\mathrm{FN}}
$$


TABLE 1 | Cross-validation performances of AAC with different classifiers on PM275.

\begin{tabular}{lcccccc}
\hline Classifier & Accuracy & Sensitivity & Precision & Recall & F-score & MCC \\
\hline LR & $\mathbf{8 2 . 9 1 \%}$ & $12.73 \%$ & $\mathbf{7 1 . 2 6} \%$ & $56.36 \%$ & $\mathbf{5 5 . 2 8} \%$ & $\mathbf{0 . 2 4}$ \\
NB & $57.82 \%$ & $\mathbf{5 8 . 7 3} \%$ & $58.16 \%$ & $\mathbf{5 8 . 0 2} \%$ & $50.68 \%$ & 0.12 \\
SVM & $80.36 \%$ & $1.82 \%$ & $40.18 \%$ & $50 \%$ & $44.56 \%$ & 0
\end{tabular}

The bold values are the maximum value for each column.

$$
\begin{gathered}
\text { Sensitivity }=\frac{\mathrm{TP}}{\mathrm{TP}+\mathrm{FN}} \\
\text { precision }=\frac{\mathrm{TP}}{\mathrm{TP}+\mathrm{FP}} \\
\text { recall }=\frac{\mathrm{TN}}{\mathrm{TN}+\mathrm{FP}} \\
F-\text { score }=\frac{2 * \text { precision } * \text { recall }}{\text { precision }+ \text { recall }} \\
M C C=\frac{\mathrm{TP} \times \mathrm{TN}-\mathrm{FP} \times \mathrm{FN}}{\sqrt{(\mathrm{TP}+\mathrm{FP})(\mathrm{TP}+\mathrm{FN})(\mathrm{TN}+\mathrm{FP})(\mathrm{TN}+\mathrm{FN})}}
\end{gathered}
$$

Here TP represents the number of mitochondrial proteins predicted correctly, FP represents the number of nonmitochondrial proteins predicted incorrectly, TN represents the number of non-mitochondrial proteins predicted correctly, FN denotes the number of mitochondrial proteins predicted incorrectly.

\section{RESULTS}

Experiments first evaluate the performances of using AAC, DPC, TPC as the features, and the different machine learning algorithms as classifiers. Results demonstrate that TPC performs better than other feature sets (Tables 1, 2). So, we use TPC as features and then use the ANOVA to select a part of TPC as features in order to improve the prediction accuracy. Experiments then evaluate the performances of using the optimized TPC as the features. Results suggest that the optimized TPC significantly improves the accuracy of discriminating mitochondrial proteins of Plasmodium, especially for the SVM classifier (Table 3). Therefore, we obtain a new mode named PM-OTC, using the SVM as the classifier and the optimized TPC as the features, to predict the mitochondrial proteins of Plasmodium. Experiments finally evaluate the performance of PM-OTC by comparing PM-OTC with other computational methods (PlasMit, PFMpred, PfMP-N25, PfMP-30, ID, and Ding). Results show PM-OTC is superior to the others (Table 4).

\subsection{Analysis of AAC on PM275}

We plot a histogram based on the frequency of each amino acid for each protein from PM275 (Figure 1) in order to analyze the differences between mitochondrial proteins and non-mitochondrial proteins. Figure 1 shows that mitochondrial

\begin{tabular}{|c|c|c|c|c|c|c|c|}
\hline $\begin{array}{l}\text { Feature } \\
\text { vector }\end{array}$ & Classifier & Accuracy & Sensitivity & Precision & Recall & F-score & MCC \\
\hline & LR & $83.27 \%$ & $33.27 \%$ & $76.59 \%$ & $64.36 \%$ & $66.84 \%$ & 0.39 \\
\hline \multirow[t]{3}{*}{ DPC } & NB & $60.73 \%$ & $86.73 \%$ & $63.55 \%$ & $70.49 \%$ & $57.46 \%$ & 0.33 \\
\hline & SVM & $86.18 \%$ & $29.45 \%$ & $92.68 \%$ & $72.73 \%$ & $68.33 \%$ & 0.49 \\
\hline & LR & $82.55 \%$ & $31.45 \%$ & $74.25 \%$ & $63.23 \%$ & $65.32 \%$ & 0.35 \\
\hline \multirow[t]{3}{*}{$\mathrm{AAC}+\mathrm{DPC}$} & NB & $60.73 \%$ & $86.73 \%$ & $63.61 \%$ & $70.48 \%$ & $57.42 \%$ & 0.33 \\
\hline & SVM & $85.82 \%$ & $27.64 \%$ & $92.52 \%$ & $63.82 \%$ & $67.18 \%$ & 0.48 \\
\hline & LR & $88.73 \%$ & $42.55 \%$ & $93.91 \%$ & $71.27 \%$ & $75.54 \%$ & 0.60 \\
\hline \multirow[t]{2}{*}{ TPC } & NB & $82.91 \%$ & $12.73 \%$ & $81.24 \%$ & $56.36 \%$ & $56.09 \%$ & 0.29 \\
\hline & SVM & $89.82 \%$ & $48.18 \%$ & $94.43 \%$ & $74.09 \%$ & $78.80 \%$ & 0.65 \\
\hline
\end{tabular}
proteins have more alanine, phenylalanine, glycine, isoleucine, leucine, proline, glutamine, arginine, threonine, and valine
TABLE 2 | Cross-validation performances of DPC, TPC, and combination features using different classifiers on PM275.

The bold values are the maximum value for each column.

TABLE 3 | Cross-validation performances of optimized TPC using different classifiers on PM275.

\begin{tabular}{lccccccc}
\hline Classifier & $\begin{array}{c}\text { Feature } \\
\text { dimension }\end{array}$ & Accuracy Sensitivity Precision & Recall & F-score & MCC \\
\hline LR & 984 & $91.27 \%$ & $55.64 \%$ & $95.13 \%$ & $77.82 \%$ & $82.8 \%$ & 0.71 \\
NB & 2578 & $85.09 \%$ & $24 \%$ & $92.22 \%$ & $62 \%$ & $63.98 \%$ & 0.43 \\
SVM & 399 & $\mathbf{9 4 . 9 1 \%}$ & $\mathbf{7 4 . 1 8 \%}$ & $\mathbf{9 7 . 0 5 \%}$ & $\mathbf{8 7 . 0 9 \%}$ & $\mathbf{9 0 . 8 6 \%}$ & $\mathbf{0 . 8 3}$
\end{tabular}

The bold values are the maximum value for each column.

TABLE 4 | Cross-validation performance of PM-OTC compared with other methods on PfM175.

\begin{tabular}{lcccc}
\hline Method & Accuracy & Sensitivity & Recall & MCC \\
\hline PlasMit (Bender et al., 2003) & $90.00 \%$ & $94.00 \%$ & $89.00 \%$ & 0.74 \\
PFMpred (Verma et al., 2010) & $92.00 \%$ & $97.50 \%$ & $90.40 \%$ & 0.81 \\
PfMP-N25 (Jia et al., 2011) & $96.00 \%$ & $87.50 \%$ & 98.50 & 0.93 \\
PfMP-30 (Jia et al., 2011) & $98.80 \%$ & $97.50 \%$ & $\mathbf{9 9 . 3 0 \%}$ & 0.97 \\
ID (Chen et al., 2012) & $92.00 \%$ & $\mathbf{1 0 0 \%}$ & $89.63 \%$ & 0.82 \\
Ding (Ding and Li, 2015) & $\mathbf{9 7 . 1 0 \%}$ & $90.00 \%$ & $\mathbf{9 9 . 3 0 \%}$ & 0.92 \\
Our method & $\mathbf{9 9 . 4 3 \%}$ & $97.50 \%$ & $98.75 \%$ & $\mathbf{0 . 9 8}$
\end{tabular}

The bold values are the maximum value for each column.

than non-mitochondrial proteins. On the contrary, nonmitochondrial proteins have more aspartic, glutamic, lysine, asparagine, serine, and tyrosine than mitochondrial proteins. Only the amino acid cysteine and histidine are no significant differences in mitochondrial and non-mitochondrial proteins. We further research the prediction performance when using the $\mathrm{AAC}$ as the features. So, we extract the AAC (Equation 1) for each protein from PM275. Here we use the SVM, the Logistic Regression (short for LR) and the Naïve Bayes (short for NB) as classifiers.

Table 1 shows the results. The results show that the LR has a more excellent performance than other classifies in accuracy of $82.91 \%$, precision of $71.26 \%$, F-score of $55.28 \%$, and MCC of 0.24 . The NB performs better in sensitivity (58.73\%) and recall (58.02\%) than other classifiers. Overall, using the AAC as the features to predict mitochondrial proteins of Plasmodium have low performance. 


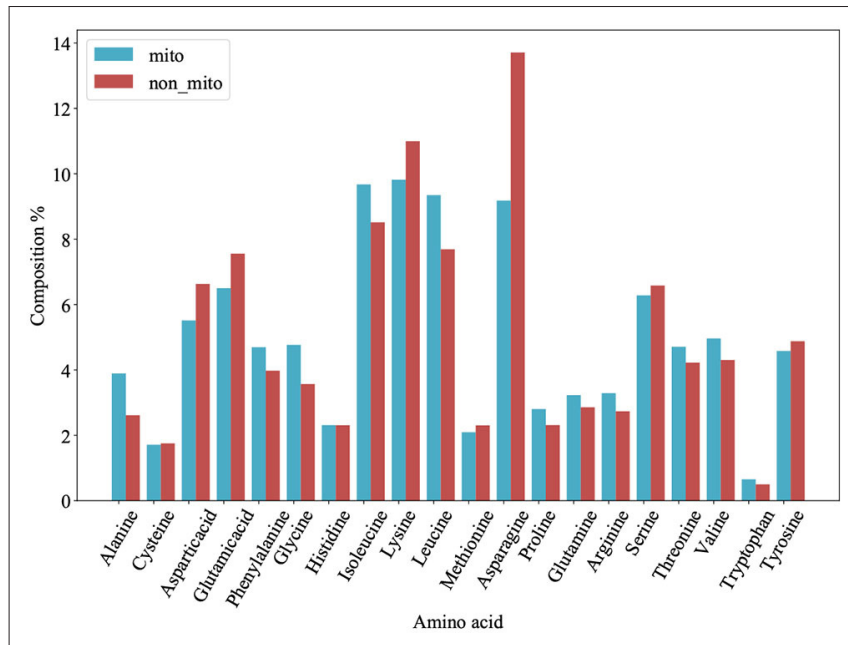

FIGURE 1 | Amino acid composition of 54 mitochondrial proteins (mito) and 221 non-mitochondrial proteins (non_mito). The abscissa represents the abbreviation of amino acid, and the ordinate represents the percentage content of amino acid.

\subsection{Prediction Performances of DPC, TPC and Combined Feature on PM275}

Next, we consider three feature sets: DPC (Equations 2, 3), DPC combined with AAC, and TPC (Equations 4, 5). We input these three feature sets into three classifiers (LR, NB, SVM). Results are recorded in Table 2. Table 2 shows that the model using the SVM as the classifier and the TPC as the features performs better in almost all measures than other models, and achieves the accuracy of $89.82 \%$, precision of $94.43 \%$, recall of $74.09 \%$, F-score of $78.80 \%$, and MCC of 0.65 . The models using TPC as the features get better performances compared with DPC and AAC+DPC. Thus, TPC has obvious advantages in discriminating mitochondrial proteins of Plasmodium. Table 1 shows that the SVM classifier does not perform as well as the other two classifiers when using the AAC as the features. However, as we can see from Table 2, the SVM classifier performs efficient and powerful when using high dimensional feature sets as the input features. So, we choose TPC as the features.

\subsection{Prediction Performance of Optimized TPC on PM275}

We use ANOVA and IFS strategy to reduce the dimension of TPC and further obtain the optimized TPC as the features. We rank the 8000 dimensional TPC according their F-value (Equation 6) and adopt IFS to generate 8000 subsets. Then we input all 8000 subsets into three classifiers (LR, NB, SVM) and calculate the accuracy of 5-fold cross-validation of each subset. Figure 2 shows the IFS curve. From Figure 2, we can see that the accuracy of 5-fold cross-validation has the maximum $94.91 \%$ when using the SVM as the classifier. And the optimized TPC only contains 399 tripeptides. Table 3 shows that the model using the SVM as the classifier and the optimized TPC as the features performs more reliable than the other classifiers. Accordingly, the PMOTC model uses the top 399 ranked tripeptides as features and

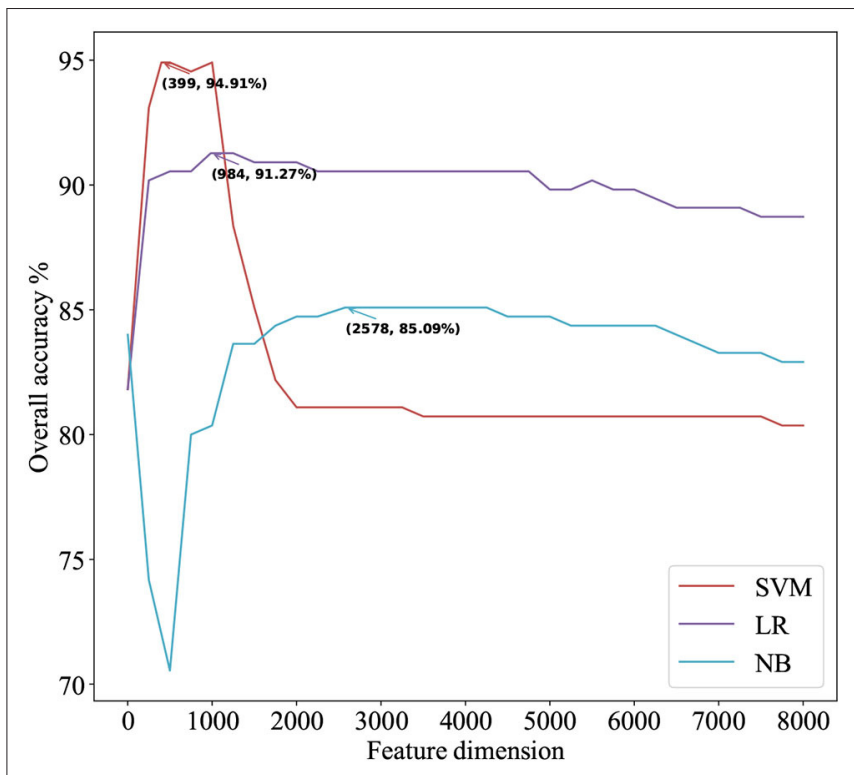

FIGURE 2 | The IFS curve for predicting mitochondrial proteins of Plasmodium using three classifiers. The accuracies of the SVM classifier and the LR classifier improve when the number of features is initially increased. When the number of features exceeds 399, the accuracy of the SVM classifier decreases significantly and finally returns to stable. With the increase in the number of features, the accuracy of the NB classifier first decreases significantly and then gradually increases to stable.

the SVM as the classifier to predict the mitochondrial proteins of Plasmodium. Figure 3 shows the structure of PM-OTC.

Table 2 shows that the model using the SVM as the classifier and the TPC as features can only achieve the crossvalidated accuracy by $89.82 \%$, which is lower than that $(94.91 \%)$ of PM-OTC. So, the optimized TPC significantly enhances the prediction performance of the model using SVM as the classifier, especially in accuracy, and improves it by $5.09 \%$. This result shows that the original 8000 dimensional TPC includes redundant or noise information. Table 2 shows that the modes using the LR and NB as the classifiers and the optimized TPC as the features also improve the prediction performance (Figure 3 ). This result shows that the optimized TPC is an effective and efficient feature vector in predicting mitochondrial proteins of Plasmodium.

\subsection{Performance of the PM-OTC on PfM175}

Most of the published methods can only predict mitochondrial proteins of $P$. falciparum and built on PfM175. Accordingly, we train and test PM-OTC adopting 5-fold cross-validation on PfM175 in order to compare our approach with other computational methods. Table 4 records the comparison of all methods. Result shows that our method outperforms other methods with an accuracy of $99.43 \%$ and MCC of 0.98. Meanwhile, precision and F-score of our method are 99.64 and $99.15 \%$, respectively. This result indicates that the 


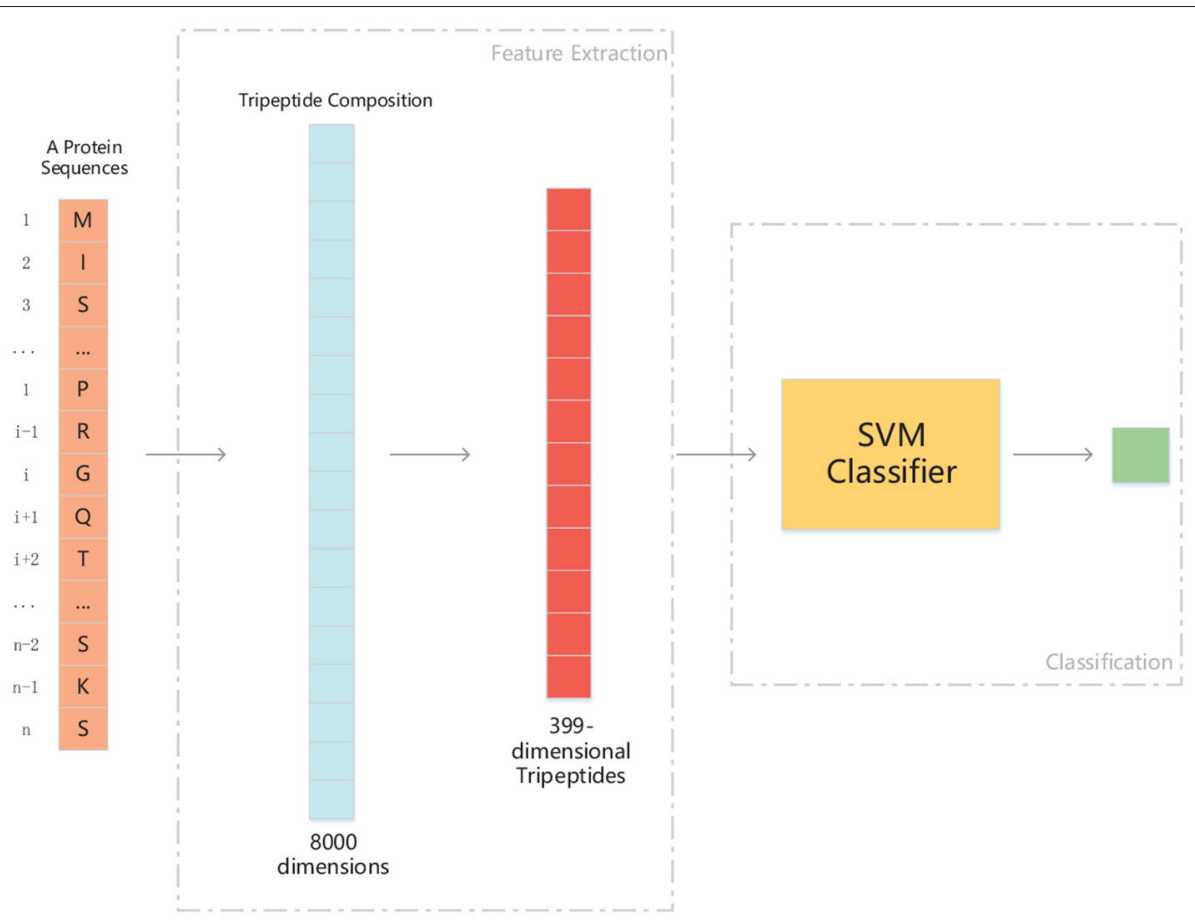

FIGURE 3 | The structure of PM-OTC model. The input data is whole protein sequence. First, through extracting tripeptides from raw sequence, 8000-dimensional TPC are obtained. And then, TPC constitute a feature vector of 399 dimensions by ANOVA, which is fed into the SVM classifier for prediction.

prediction results of PM-OTC are more correct and reliable than other approaches.

\section{CONCLUSION}

Predicting mitochondrial proteins of Plasmodium is the key to treating malaria because mitochondrion is a suitable target for anti-malarial drugs. Here we build the PM-OTC to predict the mitochondrial proteins of Plasmodium instead of only predicting mitochondrial proteins of $P$. falciparum.

The PM-OTC uses the optimized TPC as the features and the SVM as the classifier to predict mitochondrial proteins of Plasmodium. The performance of PM-OTC on PM275 indicates that PM-OTC performs well in predicting mitochondrial proteins of Plasmodium with an accuracy of $94.91 \%$. The performance of PM-OTC on PfM175 shows that PM-OTC improves the accuracy by $0.64-9.43 \%$ compared with other methods. So, the PM-OTC is efficient and effective in predicting mitochondrial proteins of $P$. falciparum.

\section{REFERENCES}

Ahmad, K., Waris, M., and Hayat, M. (2016). Prediction of protein submitochondrial locations by incorporating dipeptide composition into chou's general pseudo amino acid composition. J. Membr. Biol. 249, 293-304. doi: $10.1007 / \mathrm{s} 00232-015-9868-8$

Altschul, S. F., Madden, T. L., Schäffer, A. A., Zhang, J., Zhang, Z., Webb, M., et al. (1997). Gapped blast and psi-blast: a new generation of protein database

\section{DATA AVAILABILITY STATEMENT}

All datasets presented in this study are included in the article/supplementary material.

\section{SOFTWARE AVAILABLE}

The software of PM-OTC can download from https://github. com/CS-BhD/PMOTC.

\section{AUTHOR CONTRIBUTIONS}

HB proposed the method. HB and JW designed the experiments. All author wrote the manuscript.

\section{FUNDING}

This work was supported by the National Natural Science Foundation of China [61661040].

search programs. Nucleic Acids Res. 25, 3389-3402. doi: 10.1093/nar/25. 17.3389

Amari, S.-I., and Wu, S. (1999). Improving support vector machine classifiers by modifying kernel functions. Neural Netw. 12, 783-789. doi: 10.1016/S0893-6080(99)00032-5

Anderson, M. J. (2001). A new method for non-parametric multivariate analysis of variance. Aust. Ecol. 26, 32-46. doi: 10.1111/j.1442-9993.2001.01 070.pp.x 
Bender, A., van Dooren, G. G., Ralph, S. A., McFadden, G. I., and Schneider, G. (2003). Properties and prediction of mitochondrial transit peptides from Plasmodium falciparum. Mol. Biochem. Parasitol. 132, 59-66. doi: 10.1016/j.molbiopara.2003.07.001

Bhasin, M., and Raghava, G. (2004a). Gpcrpred: an svm-based method for prediction of families and subfamilies of g-protein coupled receptors. Nucleic Acids Res. 32(Suppl_2), W383-W389. doi: 10.1093/nar/gkh416

Bhasin, M., and Raghava, G. P. S. (2004b). Eslpred: Svm-based method for subcellular localization of eukaryotic proteins using dipeptide composition and psi-blast. Nucleic Acids Res. 32, W414-W419. doi: 10.1093/nar/gkh350

Chen, Y.-L., Li, Q.-Z., and Zhang, L.-Q. (2012). Using increment of diversity to predict mitochondrial proteins of malaria parasite: integrating pseudoamino acid composition and structural alphabet. Amino Acids 42, 1309-1316. doi: 10.1007/s00726-010-0825-7

Chou, K. (2005). Using amphiphilic pseudo amino acid composition to predict enzyme subfamily classes. Bioinformatics 21, 10-19. doi: 10.1093/bioinformatics/bth466

Ding, C. H., and Dubchak, I. (2001). Multi-class protein fold recognition using support vector machines and neural networks. Bioinformatics 17, 349-358. doi: 10.1093/bioinformatics/17.4.349

Ding, H., and Li, D. (2015). Identification of mitochondrial proteins of malaria parasite using analysis of variance. Amino Acids 47, 329-333. doi: 10.1007/s00726-014-1862-4

Fu, L., Niu, B., Zhu, Z., Wu, S., and Li, W. (2012). Cd-hit: accelerated for clustering the next-generation sequencing data. Bioinformatics 28, 3150-3152. doi: 10.1093/bioinformatics/bts565

Gardner, M. J., Hall, N., Fung, E., White, O., Berriman, M., Hyman, R. W., et al. (2002). Genome sequence of the human malaria parasite Plasmodium falciparum. Nature 419, 498-511. doi: 10.1038/nature01097

Guo, Y., Yu, L., Wen, Z., and Li, M. (2008). Using support vector machine combined with auto covariance to predict protein-protein interactions from protein sequences. Nucleic Acids Res. 36, 3025-3030. doi: 10.1093/nar/gkn159

Hofmann, T., Schölkopf, B., and Smola, A. J. (2008). Kernel methods in machine learning. Ann. Stat. 36, 1171-1220. doi: 10.1214/009053607000000677

Hua, S., and Sun, Z. (2001). Support vector machine approach for protein subcellular localization prediction. Bioinformatics 17, 721-728. doi: 10.1093/bioinformatics/17.8.721

Jia, C., Liu, T., Chang, A. K., and Zhai, Y. (2011). Prediction of mitochondrial proteins of malaria parasite using bi-profile bayes feature extraction. Biochimie 93, 778-782. doi: 10.1016/j.biochi.2011.01.013

Karchin, R., Karplus, K., and Haussler, D. (2002). Classifying g-protein coupled receptors with support vector machines. Bioinformatics 18, 147-159. doi: 10.1093/bioinformatics/18.1.147

Kumar, M., Verma, R., and Raghava, G. P. S. (2006). Prediction of mitochondrial proteins using support vector machine and hidden markov model. J. Biol. Chem. 281:5357. doi: 10.1074/jbc.M511061200

Kumar, R., Kumari, B., and Kumar, M. (2018). Proteome-wide prediction and annotation of mitochondrial and sub-mitochondrial proteins by incorporating domain information. Mitochondrion 42, 11-22. doi: 10.1016/j.mito.2017.10.004

Liao, B., Jiang, J.-B., Zeng, Q.-G., and Zhu, W. (2011). Predicting apoptosis protein subcellular location with pseaac by incorporating tripeptide composition. Protein Peptide Lett. 18, 1086-1092. doi: 10.2174/092986611797200931

Lin, H., Ding, C., Yuan, L.-F., Chen, W., Ding, H., Li, Z.-Q., et al. (2013). Predicting subchloroplast locations of proteins based on the general form of chou's pseudo amino acid composition: approached from optimal tripeptide composition. Int. J. Biomath. 6:1350003. doi: 10.1142/S1793524513500034

Murakami, Y., and Mizuguchi, K. (2010). Applying the naïve bayes classifier with kernel density estimation to the prediction of protein-protein interaction sites. Bioinformatics 26, 1841-1848. doi: 10.1093/bioinformatics/btq302
Nakashima, H., and Nishikawa, K. (1994). Discrimination of intracellular and extracellular proteins using amino acid composition and residue-pair frequencies. J. Mol. Biol. 238, 54-61. doi: 10.1006/jmbi.1994.1267

Pedregosa, F., Varoquaux, G., Gramfort, A., Michel, V., Thirion, B., Grisel, O., et al. (2011). Scikit-learn: Machine learning in Python. J. Mach. Learn. Res. 12, 2825-2830. doi: 10.1524/auto.2011.0951

Phillips, M. A., Burrows, J. N., Manyando, C., Huijsduijnen, R. H. V., Van Voorhis, W. C., and Wells, T. N. C. (2017). Malaria. Nat. Rev. Dis. Primers 3:17050. doi: $10.1038 /$ nrdp. 2017.50

Qu, K., Wei, L., and Zou, Q. (2019). A review of dna-binding proteins prediction methods. Curr. Bioinform. 14, 246-254. doi: 10.2174/1574893614666181212102030

Rashid, M., Saha, S., and Raghava, G. P. (2007). Support vector machinebased method for predicting subcellular localization of mycobacterial proteins using evolutionary information and motifs. Bmc Bioinformatics 8:337. doi: $10.1186 / 1471-2105-8-337$

Rish, I. (2001). "An empirical study of the naive bayes classifier," in IJCAI 2001 Workshop on Empirical Methods in Artificial Intelligence Vol. 3, 41-46.

Shen, H., and Chou, K. (2006). Ensemble classifier for protein fold pattern recognition. Bioinformatics 22, 1717-1722. doi: 10.1093/bioinformatics/btl170

Vaidya, A. B., and Mather, M. W. (2009). Mitochondrial evolution and functions in malaria parasites. Annu. Rev. Microbiol. 63, 249-267. doi: 10.1146/annurev.micro.091208.073424

Vapnik, V. N. (2000). The Nature of Statistical Learning Theory. Springer.

Verma, R., Varshney, G. C., and Raghava, G. (2010). Prediction of mitochondrial proteins of malaria parasite using split amino acid composition and pssm profile. Amino Acids 39, 101-110. doi: 10.1007/s00726-009-0381-1

Wan, S., Mak, M.-W., and Kung, S.-Y. (2015). mplr-loc: an adaptive decision multi-label classifier based on penalized logistic regression for protein subcellular localization prediction. Anal. Biochem. 473, 14-27. doi: 10.1016/j.ab.2014.10.014

Wei, L., Liao, M., Gao, X., and Zou, Q. (2015). Enhanced protein fold prediction method through a novel feature extraction technique. IEEE Trans. Nanobiosci. 14, 649-659. doi: 10.1109/TNB.2015.2450233

Wei, L., Tang, J., and Zou, Q. (2017). Local-dpp: an improved dna-binding protein prediction method by exploring local evolutionary information. Inform. Sci. 384, 135-144. doi: 10.1016/j.ins.2016.06.026

Zavaljevski, N., Stevens, F. J., and Reifman, J. (2002). Support vector machines with selective kernel scaling for protein classification and identification of key amino acid positions. Bioinformatics 18, 689-696. doi: 10.1093/bioinformatics/18.5.689

Zeng, M., Zhang, F., Wu, F.-X., Li, Y., Wang, J., and Li, M. (2019). Protein-protein interaction site prediction through combining local and global features with deep neural networks. Bioinformatics 36, 1114-1120. doi: 10.1093/bioinformatics/btz699

Zou, Q. (2019). Latest machine learning techniques for biomedicine and bioinformatics. Curr. Bioinform. 14, 176-177. doi: $10.2174 / 157489361403190220112855$

Conflict of Interest: The authors declare that the research was conducted in the absence of any commercial or financial relationships that could be construed as a potential conflict of interest.

Copyright (c) 2020 Bian, Guo and Wang. This is an open-access article distributed under the terms of the Creative Commons Attribution License (CC BY). The use, distribution or reproduction in other forums is permitted, provided the original author(s) and the copyright owner(s) are credited and that the original publication in this journal is cited, in accordance with accepted academic practice. No use, distribution or reproduction is permitted which does not comply with these terms. 